\title{
Collaborative care improves health outcomes in older people with depression and arthritis
}

Lin EH, Katon W, Von Korff M, et al. Effect of improving depression care on pain and functional outcomes among older adults with arthritis: a randomized controlled trial. JAMA 2003;290:2428-9.

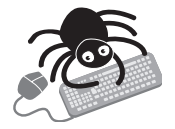

This article contains extra text on the EBMH website

Does collaborative care for depression improve pain and functional outcomes in older people with depression and arthritis?

\section{METHODS}

$\square-$

Design: Randomised controlled trial

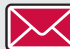

Allocation: Concealed.

Blinding: Assessors blinded to treatment.

Follow up period: 12 months.

Setting: 18 primary care clinics at 8 healthcare organisations across the United States; July 1999-August 2001.

Patients: This was a preplanned subgroup analysis of 1001 participants in the IMPACT ${ }^{1}$ trial reporting depression plus arthritis at baseline. Inclusion criteria: $>60$ years of age, current major depression or dysthymia (DSM-IV). Exclusions: history of bipolar disorder or psychosis; ongoing psychiatric treatment; severe cognitive impairment, or acute risk of suicide.

$\mathbf{R}_{\mathbf{X}}$

Intervention: People received either usual care, or the improving mood-promoting access to collaborative treatment (IMPACT) intervention. IMPACT comprised depression care management by a psychiatrist or primary care expert who provided education and support to assist people in identifying their preferred treatment (antidepressant medication or a brief psychotherapy for depression).

Outcomes: Arthritis pain intensity $10=$ no pain to $10=$ most severe pain); arthritis related interference with daily activities $10=$ no interference to $10=$ unable to perform any activities); pain related interference with daily activities (RAND short form assessment; $1=$ not at all to $5=$ extremely).

$\square$ Patient follow up: $96.3 \%$

\section{MAIN RESULTS}

In people with depression and arthritis, arthritis related pain and functional outcomes significantly improved with collaborative care compared with usual care over 12 months (see web extra table).

\section{CONCLUSIONS}

In people with depression and arthritis, collaborative care management improves arthritis related pain and functional outcomes compared with usual care.

For correspondence: Elizabeth Lin, Center for Health Studies, Group Health Cooperative, Seattle, Washington, USA; lin.e@ghc.org

Sources of funding: Wyeth, Eli Lilly, Pfizer, Merck \& Co, and Solvay.
1. Unutzer J, Katon W, Callahan CM, et al. IMPACT Investigators. Collaborative care management of late-life depression in the primary care setting: a randomized controlled trial. JAMA 2002;288:2836-45.

Commentary

D epression is a major cause of disability worldwide. Evidence for the effectiveness of various pharmacological and psychological treatments is abundant, yet outcomes are often disappointing. This may reflect poor patient understanding of the illness, and poor concordance with antidepressant medication. Recognition of these problems has led to growing interest in the development and evaluation of pragmatic, multifaceted, patient focused interventions, often termed "collaborative care". ${ }^{1}$ Encouraging evidence exists that such interventions are effective for people with depression, ${ }^{2}$ but there is a dearth of evidence for people with depression and comorbid physical disorders.

Evidence is emerging of the effectiveness of psychological and psychopharmacological treatments in people with arthritis, whether or not they have depression. Several randomised controlled trials demonstrate analgesic effects of antidepressants in people with arthritis. ${ }^{3} \mathrm{~A}$ recent randomised controlled trial of a cognitive-behavioural intervention in people with recent onset rheumatoid arthritis demonstrates significant reductions in both physical and psychological morbidity. ${ }^{4}$

Higher rates of depression are associated with many common physical disorders, including arthritis. ${ }^{5}$ Extending the principles of collaborative care to people with depression and comorbid physical illness therefore makes good sense. Lin et al's study extends the already convincing evidence that, in the care of depression, it's not just what you prescribe, it's also the way that you prescribe, educate the patient, and monitor compliance and clinical outcomes. Importantly, depressive symptoms, disability, and pain were all significantly improved at 12 months.

Despite this encouraging result, it is early days for the availability of robust evidence of effectiveness of collaborative care for the specific group of older people with arthritis. This study needs urgent replication in the UK and elsewhere. Rigorous evaluation of the cost effectiveness of such interventions is also needed, as hard pressed service providers are otherwise unlikely to provide the necessary resources.

In the meantime, it would be prudent for clinicians and health service managers to ensure that there are effective and reliable systems of care in place to identify and manage people with physical disorders and comorbid depression, whenever the required resources already exist. Dr Jonathan Price, MA DPhil MRCPsych Department of Psychiatry, University of Oxford, Oxford, UK

1 Katon W, Von Korff M, Lin E, et al. Rethinking practitioner roles in chronic illness: the specialist primary care physician and the practice nurse. Gen Hosp Psychiatry 2001;23:138-44.

2 Gilbody S, Whitty P, Grimshaw J, et al. Educational and organizational interventions to improve the management of depression in primary care. A systematic review. JAMA 2003:289:3145-51.

3 Fishbain D. Evidence-based data on pain relief with antidepressants. Ann Medicine 2000;32:305-16.

4 Sharpe L, Sensky T, Timberlake N, et al. A blind, randomized, controlled trial of cognitive-behavioural intervention for patients with recent onset rheumatoid arthritis: preventing psychological and physical morbidity. Pain $2001 ; 89: 275-83$.

5 Katz P, Yelin E. Prevalence and correlates of depressive symptoms among persons with rheumatoid arthritis. J Rheumatol 1993;20:790-6. 